\title{
Levetiracetam Serum Concentrations in Pediatric Patients: Is There a Role in Clinical Decision Making?
}

Müjgan Arslan ( $\nabla$ mujganarslan@yahoo.com )

Suleyman Demirel Universitesi Tip Fakultesi https://orcid.org/0000-0002-0486-3431

\section{Murat Yilmaz}

Suleyman Demirel Universitesi Tip Fakultesi

Adnan Karaibrahimoğlu

Suleyman Demirel Universitesi Tip Fakultesi

\section{Research Article}

Keywords: Levetiracetam, drug monitoring, epilepsy, pediatric

Posted Date: May 18th, 2021

DOl: https://doi.org/10.21203/rs.3.rs-520130/v1

License: (c) (i) This work is licensed under a Creative Commons Attribution 4.0 International License.

Read Full License

Version of Record: A version of this preprint was published at Archives of Epilepsy on April 5th, 2022. See the published version at https://doi.org/10.54614/ArchEpilepsy.2022.77045. 


\section{Abstract}

Monitoring of levetiracetam is not frequently used in clinical practice. This study evaluated the LEV blood level concentrations, correlation with dose, and co-medication effect to decide the need for blood concentration monitoring in pediatric patients.

The children with epileptic seizures on levetiracetam therapy, aged one month-18 years, were enrolled and evaluated for gender, age, body weight, daily drug dose, comedication, and drug concentration records.

$57.9 \%$ of one hundred and forty patients were on monotherapy. The mean dose of LEV was 35.40 $\mathrm{mg} / \mathrm{kg} / \mathrm{day}$, while the mean drug concentration was $14.06 \mu \mathrm{g} / \mathrm{mL}$. The correlation between the dose and the serum concentration in the polytherapy group was poorly significant, whereas it was positive and highly significant in the monotherapy group. The mean drug concentration was within the established reference range, but concentration monitoring revealed children with serum concentrations below and above the therapeutic range. All cases in our study had the typical range of drug dose per body weight with no side effects.

Conclusion: We point out that monitoring is not practical for most patients. Due to its tolerability over an extensive concentration range, the clinical assessment remains the monitoring strategy for patients with epilepsy on LEV therapy.

\section{What Is Known}

- The interactions between levetiracetam and concomitant antiepileptic drugs is unpredictable.

- Therapeutic drug monitoring contribute to managing patients on polypharmacy.

\section{What Is New}

- Levetiracetam has wide therapeutic range, predictable serum concentration changes, possible minimal drug interaction, and low prevalence of side-effects.

- Therapeutic drug monitoring is unnecessary for optimizing levetiracetam therapy.

\section{Introduction}

The treatment of epilepsy requires individualizing drug doses to increase efficacy while avoiding adverse events. In clinical practice, the treatment of epilepsy is based on effectiveness and tolerability. However, clinical effects can be anticipated by measuring antiepileptic drug (AED) serum concentrations [1]. Drug monitoring is often recommended in pediatric patients for certain AEDs due to significant interindividual differences and unpredictable drug disposition [2]. Levetiracetam (LEV) is a second-generation broadspectrum AED, effective in threatening multiple seizure types in children. The lack of effect on cytochrome P450 and its minimal protein binding reduce its pharmacokinetic interactions. In patients receiving LEV, blood level monitoring is not frequently used in clinical follow-up due to its favorable 
pharmacokinetics and the lack of reference for the drug's therapeutic range [3-5]. This study evaluated the LEV blood level concentrations, correlation with dose, and co-medication effect to decide the need for blood concentration monitoring in pediatric patients.

\section{Methods}

The institutional ethics committee approved this retrospective study (159-22.05.2020). LEV serum concentrations were determined using a kit with high-performance liquid chromatography in a single laboratory. We retrospectively searched the institution's database for children with epileptic seizures on LEV treatment as monotherapy or polytherapy between January 2018-January 2020. A hundred and forty children with epilepsy aged one month-18 years were enrolled in the study. They presented different types of epilepsy syndromes. In the management of epileptic patients, the drug serum concentration is determined a month after treatment initiation or a month after a change in dosage. We evaluated the patient's gender, age, body weight, daily drug dose, comedication, and drug blood level records. Patients on LEV were divided into two groups; monotherapy and polytherapy group.

\section{Statistical Analysis}

Descriptive statistics were presented as frequency (percentage) for categorical variables and mean $\pm S D$ (median, min, max where necessary) for numerical variables. Chi-square test with Monte Carlo Exact method was used to determine the relations between the categorical variables. Mann-Whitney $U$ test was used to compare drug groups since the distribution of the continuous variables was not normal by the Shapiro-Wilk test. Spearman's Rho correlation coefficients were calculated to see the relations between the dose and the drug's serum level. The associations were visualized by scatter plots. $p<0.05$ value was considered a statistically significant result for $5 \%$ type-l error in all analyses. The study's comments were performed by SPSS 20.0 (IBM Inc, Chicago, IL, USA) software.

\section{Power Analysis}

The apriori power analysis was performed by GPower 9.1.2 software. The effect size was calculated as 0.58 using the dose level of LEV in a pilot study. Choosing a one-tailed t-test for the Mann-Whitney U test for independent samples, the sample size was $54 \& 76$ for each group (total 130). We considered the power as 0.80 , the error as $5 \%$, and the allocation ratio as 1.5 .

\section{Results}

One hundred forty epilepsy patients, aged between 1 and 18 years, were included in the study. $53.6 \%$ of the patients were girls, and the mean age was $9.45 \pm 4.91$ years. Eighty-one $(57.9 \%)$ of the patients were on monotherapy. The others were on polytherapy. The most commonly used concomitant drugs were valproate, clobazam, carbamazepine. We found that the mean dose of LEV was $35.40 \pm 16.53$ (10.2$77.0) \mathrm{mg} / \mathrm{kg} /$ day, while the mean drug level in blood was $14.06 \pm 11.14(0.1-55.3) \mu \mathrm{g} / \mathrm{mL}$. A significant and positive correlation was found between the drug dose and the drug level in the blood. $(R=0.484 ; p<$ 
0.001) (Table 1). The scatter plot showed the correlation between the daily dose and the medication's serum level in Fig. 1. 
Table 1

General characterisics of the patients.

\begin{tabular}{|c|c|c|c|c|}
\hline Characteristics & Categories & $\mathrm{n}(\%)$ & & \\
\hline \multirow[t]{2}{*}{ Gender } & Female & $75(53.6)$ & & \\
\hline & Male & $65(46.4)$ & & \\
\hline \multirow[t]{2}{*}{ Comedication } & Polytherapy & $59(42.1)$ & & \\
\hline & Monotherapy & $81(57.9)$ & & \\
\hline \multirow{17}{*}{$\begin{array}{l}\text { Number of } \\
\text { antiepileptics }\end{array}$} & $L E V$ & 81 & & \\
\hline & $L E V, V P A$ & 19 & & \\
\hline & $\angle E V, C B Z$ & 5 & & \\
\hline & $L E V, P B$ & 2 & & \\
\hline & $\angle E V, O X C$ & 1 & & \\
\hline & $L E V, C L Z$ & 1 & & \\
\hline & $\angle E V, V P A, C L B$ & 9 & & \\
\hline & $L E V, V P A, P B$ & 3 & & \\
\hline & $\angle E V, V P A, T P M$ & 5 & & \\
\hline & $\angle E V, C B Z, C L B$ & 1 & & \\
\hline & $\angle E V, L T G, C L Z$ & 1 & & \\
\hline & $\angle E V, T P M, O X C$ & 1 & & \\
\hline & $\angle E V, O X C, Z N S$ & 1 & & \\
\hline & $\angle E V, C B Z, T P M$ & 1 & & \\
\hline & $\angle E V, C B Z, T P M, C L B$ & 1 & & \\
\hline & $\angle E V, C B Z, L C M$ & 1 & & \\
\hline & & mean $\pm S D$ & $\begin{array}{l}\text { median, min, } \\
\text { max }\end{array}$ & $\begin{array}{l}\text { Correlation, } R \\
\text { (p) }\end{array}$ \\
\hline Age & year & $9.45 \pm 4.91$ & $9.5,1.0-18.0$ & \\
\hline LEV dose & $\mathrm{mg} / \mathrm{kg} / \mathrm{day}$ & $\begin{array}{l}35.40 \pm \\
16.53\end{array}$ & $31.1,10.20-77.0$ & $\begin{array}{l}0.484(p< \\
0.001)\end{array}$ \\
\hline
\end{tabular}

$\mathrm{CBZ}=$ Carbamazepine, $\mathrm{CLB}=$ Clobazam, $\mathrm{CLZ}=$ Clonazepam, $\mathrm{LEV}=$ Levetiracetam, $\mathrm{LCM}=$ Lacosamide, $\mathrm{LTG}=$ Lamotrigine, $\mathrm{OXC}=\mathrm{Oxcarbazepine}, \mathrm{PB}=$ Phenobarbital,TPM $=$ Topiramate,VPA = Valproate, ZNS = Zonisamide,PC: Plasma concentration. 


\section{Characteristics}

LEV PC

\section{Categories}

$\mu \mathrm{g} / \mathrm{mL}$ $\mathrm{n}(\%)$

$14.06 \pm$

11.14
$10.29,0.11-$

55.31

$\mathrm{CBZ}=$ Carbamazepine,CLB $=$ Clobazam, $\mathrm{CLZ}=$ Clonazepam, $\mathrm{LEV}=$ Levetiracetam, $\mathrm{LCM}=$ Lacosamide, LTG = Lamotrigine, $\mathrm{OXC}=$ Oxcarbazepine, $\mathrm{PB}=$ Phenobarbital,TPM = Topiramate,VPA = Valproate, ZNS = Zonisamide,PC: Plasma concentration.

We compared the dose and the serum level of the drug in the monotherapy and polytherapy groups. The patients' age and gender did not differ significantly $(p=0.775),(p=0.634)$ between the groups. We found the dose to be considerably higher in the patients with co-medication $(p=0.001)$. The mean dose was calculated as $31.51 \pm 15.62 \mathrm{mg} / \mathrm{kg} /$ day in the monotherapy group, while it was $40.76 \pm 16.38 \mathrm{mg} / \mathrm{kg} / \mathrm{day}$ in the polytherapy group. The serum level was higher in the polytherapy group $(18.08 \pm 12.01 \mu \mathrm{g} / \mathrm{mL})$, but the difference was not significant $(p=0.485)$. The correlation value between the daily dose and the serum level in the patients with co-medication was poorly significant $(R=0.293 ; p=0.024)$, whereas the relation between the dose and serum level in the monotherapy group was positive and highly significant $(R=$ 0.619; $\mathrm{p}<0.001$ )(Table 2)(Fig. 2 and Fig. 3).

Table 2

The characteristics of the patients in monotherapy and polytherapy groups.

\begin{tabular}{|c|c|c|c|}
\hline Comedication & Polytherapy $(n=59)$ & Monotherapy ( $\mathrm{n}=\varepsilon$ & \\
\hline & mean $\pm S D$ (median, & in-max) & $\mathrm{p}$ \\
\hline Age (year) & $9.27 \pm 4.60$ & $9.58 \pm 5.14$ & 0.775 \\
\hline & $(10.0,1.0-18.0)$ & $(9.0,1.0-18.0)$ & \\
\hline LEV dose $(\mathrm{mg} / \mathrm{kg} /$ day $)$ & $40.76 \pm 16.38$ & $31.51 \pm 15.62$ & $0.001 *$ \\
\hline & $(40.0,12.0-70.5)$ & $(27.7,10.2-77.0)$ & \\
\hline LEV PC $(\mu \mathrm{g} / m L)$ & $18.08 \pm 12.01$ & $13.32 \pm 10.46$ & 0.485 \\
\hline & $(12.04,0.53-50.64)$ & $(9.32,0.10-55.31)$ & \\
\hline Correlation, R (p) & $0.293(p=0.024)$ & $0.619(p<0.001)$ & \\
\hline Gender, n (\%) & & & \\
\hline Female & $33(55.9)$ & $42(51.9)$ & 0.634 \\
\hline Male & $26(44.1)$ & 39 (48.1) & \\
\hline *: significant at $p<0.0$ & evel according to Mal & Whitney U test & \\
\hline PC: Plasma concentrat & & & \\
\hline
\end{tabular}


We compared LEV plasma level and LEV dose values according to age groups. Drug dose differed significantly according to age groups $(p<0.001)$. The drug dose value was relatively high in patients aged 0-1 year and moderate in the 7-12 age group. We observed that the dose value decreased significantly as the age increased ( $>12$ years old). Serum drug levels did not differ significantly by age groups (Table 3).

Table 3

Drug dose-drug plasma concentration values according to the age groups.

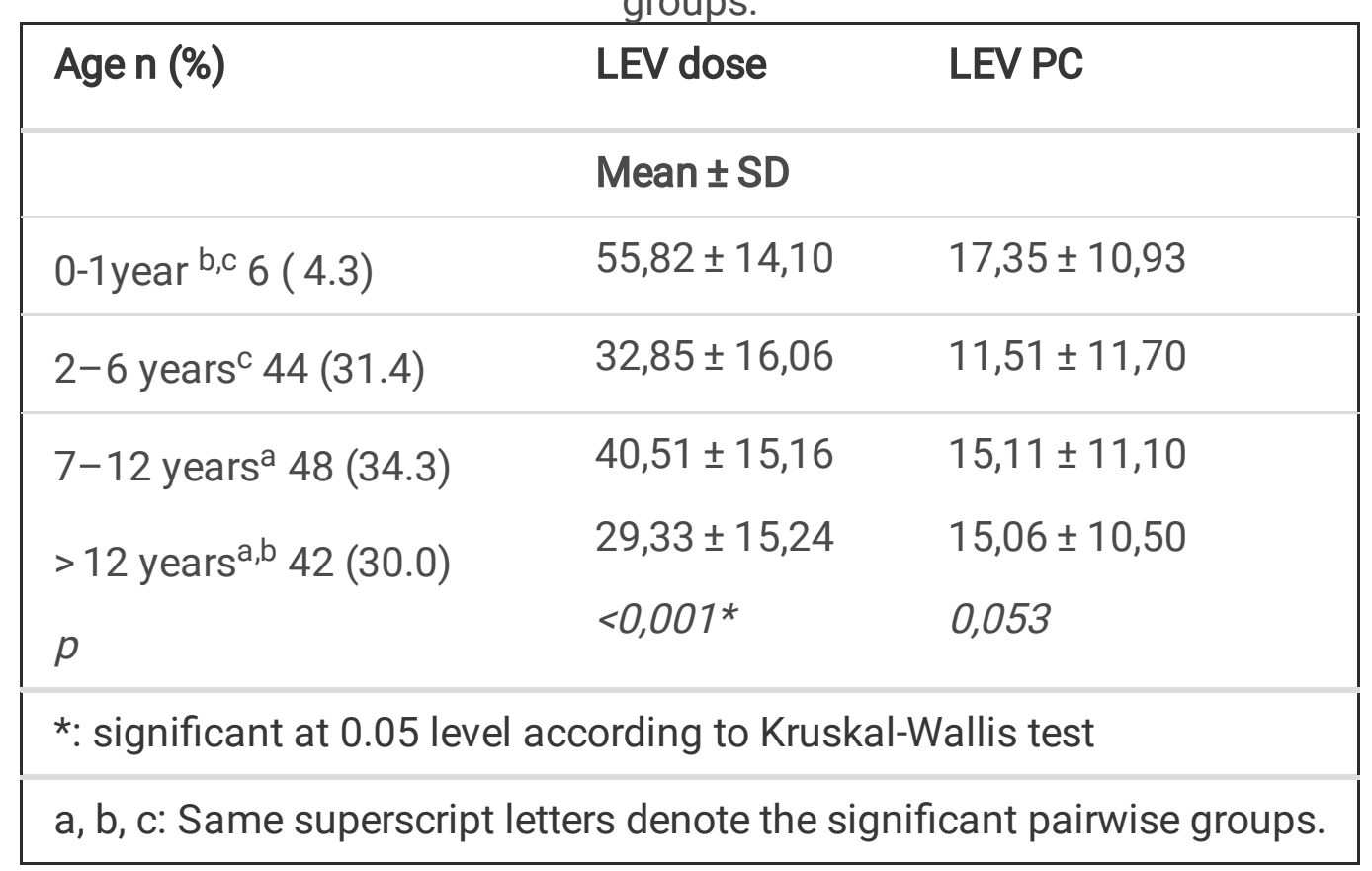

We divided patients into three groups as $<12,12-46 \mathrm{ve}>46 \mu \mathrm{g} / \mathrm{mL}$ according to the therapeutic range. Gender, age, presence of concomitant drug did not differ significantly according to plasma level groups. We found that the drug dose was significantly lower in the patient group with plasma level $<12 \mu \mathrm{g} / \mathrm{mL}$ than the other groups $(p<0.001)($ Table 4$)$. 
Table 4

Drug dose distribution according to the therapeutic range.

\begin{tabular}{|c|c|c|}
\hline LEV PC n(\%) & $\begin{array}{l}\text { LEV dose } \\
\text { (mean } \pm \text { SD) }\end{array}$ & $p$ \\
\hline$<12 \mu \mathrm{g} / \mathrm{mL} 79(56.4 \%)$ & $29.77 \pm 14.26^{+, \mathbb{Z}}$ & \multirow[t]{3}{*}{$<0,001^{*}$} \\
\hline $12-46 \mu \mathrm{g} / \mathrm{mL} 58(41.4 \%)$ & $41.88 \pm 16.37^{+}$ & \\
\hline$>46 \mu \mathrm{g} / \mathrm{mL} 3(2.1 \%)$ & $58.69 \pm 11.94^{\rrbracket}$ & \\
\hline \multicolumn{3}{|c|}{ *: significant at 0.05 level according to One-way ANOVA } \\
\hline \multicolumn{3}{|c|}{$\begin{array}{l}+, \text { same superscript symbols denote the significant pairwaise groups according to Tukey HSD test } \\
\text { with } p \text { values }<0.001 \text { and } 0.004 \text { respectively }\end{array}$} \\
\hline PC: Plasma concentration & & \\
\hline
\end{tabular}

\section{Discussion}

LEV displays linear elimination kinetics; therefore, dose changes produce predictable changes in serum concentrations [6-9], but some studies failed to show the relationship.

Sheinberg et al. reported no correlation between administered dose and serum drug level in their study even though they found no correlation between LEV serum levels and clinical efficacy [10]. Similarly, May et al. stated that the relationship between LEV dose per body weight and serum concentration deviates significantly from linearity [8].

In our study, the LEV dose and blood concentration positively and significantly correlate differently from these reports. LEV concentrations were positively correlated with dose; however, variability has been shown among patients receiving the exact dosage for body weight. May et al. explained this individual variation in parts by effects of age and co-medication [8].

As LEV is mainly given as add-on therapy, we investigated drug interaction with LEV. We divided patients into two groups; monotherapy and polytherapy. We realized that correlation was positive and highly significant, especially in the monotherapy group. The correlation value between the daily dose and the serum level in the patients with co-medication was poorly significant. This may explain the possibility of alteration in LEV concentration when concomitant antiepileptic medications are used [11].

The drug is not implicated in any drug interactions due to low hepatic metabolism, and previous studies have reported no significant interactions between LEV and other AEDs [12,13]. Nonetheless, there are studies indicating that drug interactions may still occur out. The mechanism is not well understood. The data suggest that concomitant AED, especially enzyme-inducing AED, has a moderate influence on the 
LEV kinetics by the possible increase of clearance of LEV in the presence of concomitant AED $[8,11,14-$ 16].

Mathew et al. found that patients receiving concomitant enzyme-inducing AED had drug serum concentrations lower than enzyme-inhibiting AED or no interfering AEDs [16]. Also, Stepanova et al. suggested interaction between LEV and concomitant AEDs as patients on polytherapy required a higher dose of LEV to achieve similar blood levels and provided support for the use of drug level determination [17].

In our study, the dose was significantly high in the polytherapy group, but plasma concentration did not differ between the two groups. So the data and our study results may indicate a modest effect of comedication on the LEV kinetics, informing that patients on polytherapy required a higher dose of LEV to achieve similar blood levels.

According to age groups, serum drug concentration was not different, but the dose was high in the 01years group. Young children need a higher LEV dose per weight to achieve comparable LEV concentration $[15,18]$. Pharmacokinetic differences for the drug between age groups have been described. LEV clearance during the first year of life is $60-70 \%$ higher than in adults. Thus children should receive higher LEV dosages to achieve comparable serum drug concentration [15]. May et al. found similar results and concluded that LEV dose was significantly dependant on age [8].

Studies reported that LEV concentrations were positively correlated with age, but gender had no significant impact on LEV concentration [8]. In our study, age and gender had no significant effect on LEV concentration in both groups.

Despite LEV's predictable kinetics, its target therapeutic ranges have not been well established. The International League Against Epilepsy committee set the LEV reference range for an average of $12-46$ $\mu \mathrm{g} / \mathrm{mL}$ [19]. A reference serum range of $0.11-55.31 \mu \mathrm{g} / \mathrm{mL}$ was perceived for the LEV daily dosing of $10.20-77.0 \mathrm{mg} / \mathrm{kg}$ in our study. The mean drug concentration in our patients is within the ILAE established reference range, but concentration monitoring revealed children with serum concentrations below $(56.4 \%)$ and above $3(2.1 \%)$ the therapeutic range. All cases in our study had the typical range of drug dose per body weight with no side effects.

So we point out that a range of $12-46 \mu \mathrm{g} / \mathrm{mL}$ is not practical for most patients. Due to its tolerability over an extensive concentration range, clinical assessment without measuring drug concentrations remains the monitoring strategy for patients with epilepsy on LEV therapy.

\section{Conclusions}

Because of the wide therapeutic range, predictable serum concentration changes, possible minimal drug interaction, and low prevalence of side-effects, LEV monitoring is unnecessary for optimizing drug 
therapy even in specific groups such as young age and patients on polypharmacy. LEV dosing should be guided by clinical efficacy and adverse effects outcomes, making LEV easy to use in pediatric patients.

\section{Abbreviations}

AED (antiepileptic drug)

CBZ (Carbamazepine)

CLB (Clobazam)

CLZ (Clonazepam)

LCM (Lacosamide)

LTG (Lamotrigine)

LEV (levetiracetam)

OXC (Oxcarbazepine)

PB (Phenobarbital)

PC (Plasma concentration)

TPM (Topiramate)

VPA (Valproate)

ZNS (Zonisamide)

\section{Declarations}

Funding: N/A

Conflicts of interest/Competing interests: N/A

Availability of data and material: The datasets generated during and/or analysed during the current study are available from the corresponding author on reasonable request.

Code availability: N/A

Authors' contributions:

Ethics approval: Ethical approval was waived by the local Ethics Committee of University. 
Consent to participate: Written informed consent was obtained from the parents.

Consent for publication: Written informed consent was obtained from the parents.

\section{References}

1. Patsalos PN. Clinical pharmacokinetics of levetiracetam. Clin Pharmacokinet. 2004;43:707-24.

2. Johannessen Landmark C, Baftiu A, Tysse I, et al. Pharmacokinetic variability of four newer antiepileptic drugs, lamotrigine, levetiracetam, oxcarbazepine, and topiramate: a comparison of the impact of age and comedication. Ther Drug Monit. 2012;34:440-5.

3. Das Gupta A. Therapeutic Drug Monitoring: Newer Drugs and Biomarkers. 1st ed. USA: Elsevier; 2012.

4. Krasowski MD. Therapeutic drug monitoring of the newer anti-epilepsy medications.

Pharmaceuticals. 2010;3:1909-35.

5. Touw DJ, Neef C, Thomson AH, Vinks AA. Cost-effectiveness of therapeutic drug monitoring: a systematic review. Ther Drug Monit. 2005;27:10-7.

6. Patsalos PN. Pharmacokinetic profile of levetiracetam: toward ideal characteristics. Pharmacol Ther. 2000;85(2):77-85.

7. Iwasaki T, Toki T, Nonoda Y, Ishii M. The efficacy of levetiracetam for focal seizures and its blood levels in children. Brain Dev. 2015;37(8):773-9.

8. May TW, Rambeck B, Jurgens U. Serum concentrations of Levetiracetam in epileptic patients: the influence of dose and comedication. Therapeutic Drug Monitoring. 2003;25(6) 25:690-9.

9. Giroux PC, Salas-Prato M, Theoret Y, Carmant L. Levetiracetam in children with refractory epilepsy: lack of correlation between plasma concentration and efficacy. Seizure. 2009;18(8):559-63.

10. Sheinberg R, Heyman E, Dagan Z, Youngster I, Kohn E, Gandelman-Marton R, et al. Correlation between efficacy of levetiracetam and serum levels among children with refractory epilepsy. Pediatr Neurol. 2015;52(6):624-8.

11. Perucca E, Gidal BE, Baltes E. Effects of antiepileptic comedication on levetiracetam pharmacokinetics: a pooled analysis of data from randomized adjunctive therapy trials. Epilepsy Research. 2003; 53:47- 56.

12. Patsalos PN. The pharmacokinetic characteristics of levetiracetam. Methods Find Exp Clin Pharmacol. 2003;25:123-9.

13. Radtke RA. Pharmacokinetics of levetiracetam. Epilepsia. 2001; 42(4):24-7.

14. Johannessen SI, Johannessen Landmark C. Antiepileptic drug interactions-principles and clinical implications. Curr Neuropharmacol. 2010;8(3):254-67.

15. Dahlin MG, Wide K, Ohman I. Age and comedication influence levetiracetam pharmacokinetics in children. Pediatr Neurol. 2010;43:231-5. 
16. Mathew BS, Fleming DH, Thomas M, et al. An initial experience with therapeutic drug monitoring of levetiracetam as reported from a pediatric clinical setting in India. Neurol India. 2012;60:146-9.

17. Stepanova D, Beran RG. Measurement of levetiracetam drug leves to assist with seizure control and monitoring of drug interactions with other anti-epileptic medications. Seizure. 2014;23: 371-6.

18. Leppik IE, Rarick JO, Walczak TS, et al. Effective levetiracetam doses and serum concentrations: age effects. Epilepsia. 2002;43(7):240.

19. Patsalos PN, Berry DJ, Bourgeois BFD, Cloyd JC, Glauser TA, Johannessen SI, et al. Antiepileptic drugs-best practice guidelines for therapeutic drug monitoring: a position paper by the subcommission on therapeutic drug monitoring, ILAE Commission on Therapeutic Strategies. Epilepsia. 2008;49(7): 1239-76.

\section{Figures}

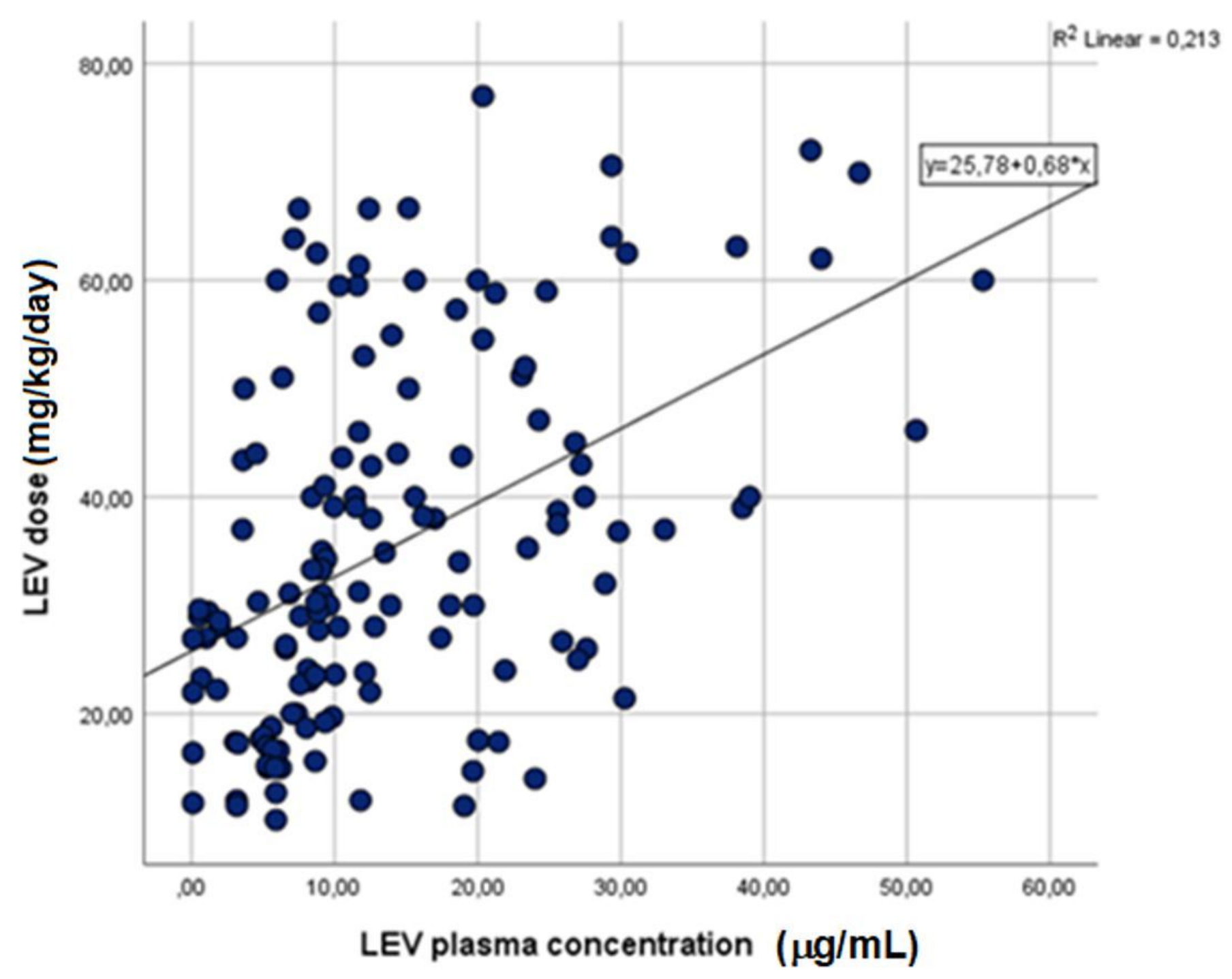

Figure 1 
The correlation between the serum level and dose of levetiracetam in all patients.

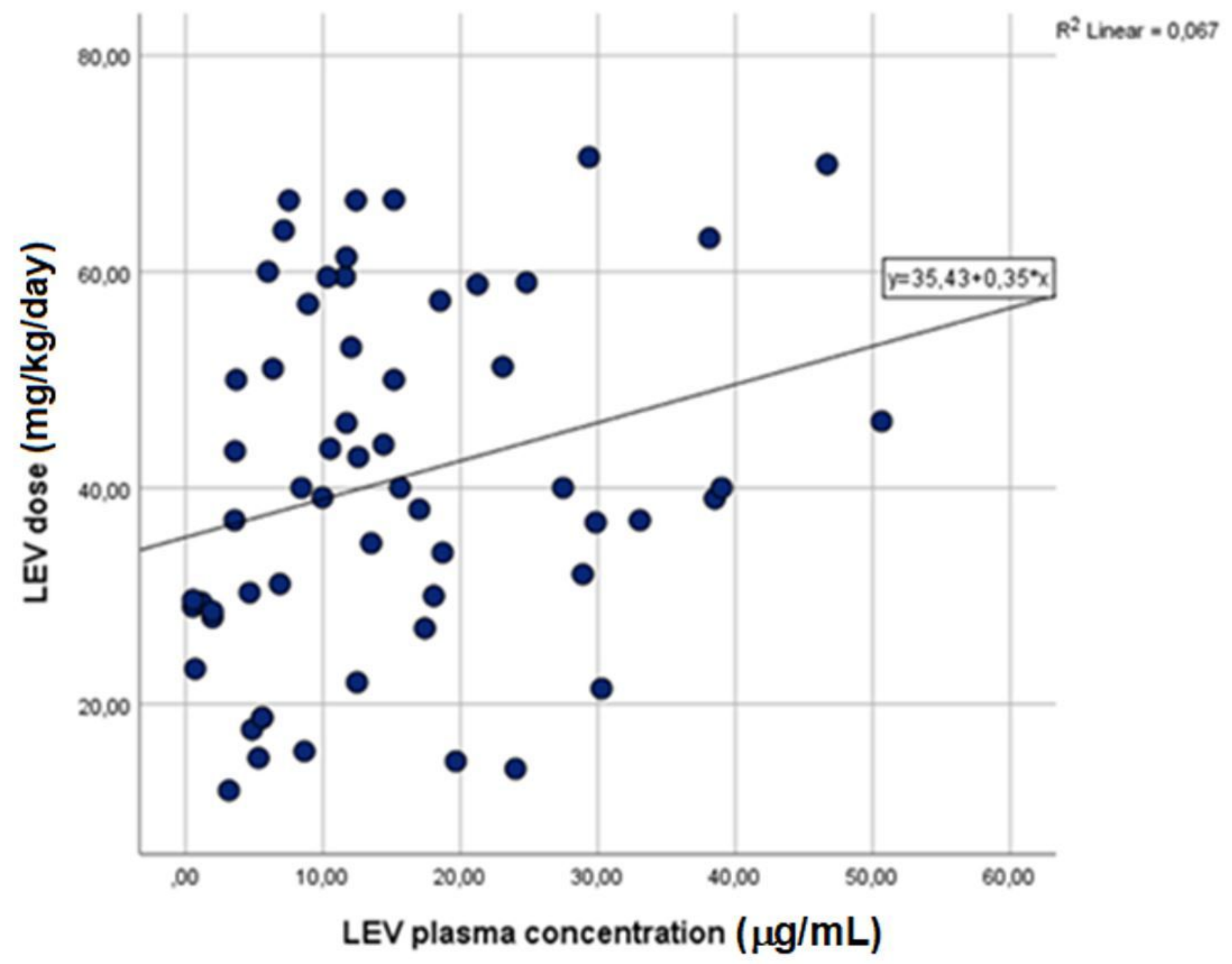

Figure 2

The correlation between the serum level and dose of levetiracetam in patients with comedication. 


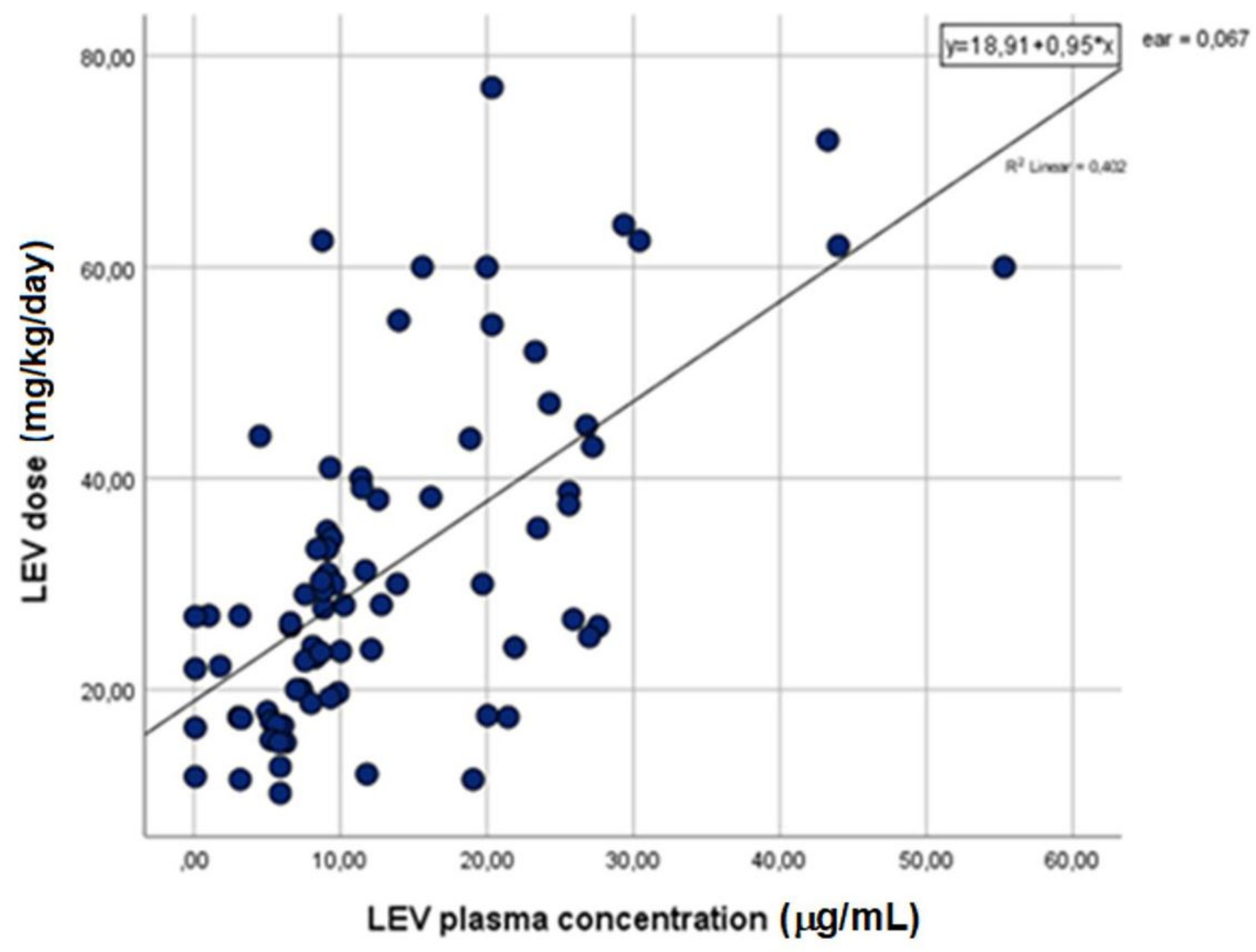

Figure 3

The correlation between the serum level and dose of levetiracetam in patients on monotherapy. 